\title{
Detección de COVID -19 (SARS-CoV-2) Mediante la Saliva: Una Alternativa Diagnóstica poco Invasiva
}

\author{
Detection of COVID-19 (SARS-CoV-2) by Saliva: A Low-invasive Diagnostic Alternative
}

\author{
Andrés Melián Rivas'; Pablo Calcumil Herrera'; Camila Boin Bakit ${ }^{2} \&$ Rolando Carrasco Soto $^{3}$
}

\begin{abstract}
MELIÁN, R. A.; CALCUMIL, H. P.; BOIN, B. C. \& CARRASCO, S. R. Detección de COVID-19 (SARS-CoV-2) mediante la saliva: Una alternativa diagnóstica poco invasiva. Int. J. Odontostomat., 14(3):316-320, 2020.

RESUMEN: La nueva enfermedad por coronavirus, también denominada COVID 19 es la última enfermedad infecciosa de preocupación internacional. Originada en Wuhan, China, se extendió a nivel mundial, resultando en la pandemia 2019-2020 y una Emergencia de Salud Pública de Preocupación Internacional (ESPII) según lo declarado por la Organización Mundial de la Salud (OMS). Esta enfermedad suele cursar con fiebre, tos, dolor de garganta, dificultad respiratoria, fatiga y malestar general, sin embargo, también se han presentado casos asintomáticos. Su diagnóstico se realiza con una combinación tanto de exámenes clínicos, radiológicos y moleculares, donde la prueba de reacción en cadena de la polimerasa con transcriptasa inversa (RT-PCR) ha sido el examen de elección para el análisis de material genético viral de muestras extraídas del tracto respiratorio superior. Se ha reportado que COVID-19 se transmite persona a persona o por contacto indirecto por gotas, lo que tiene fundamental importancia en procedimientos clínicos dentales y donde la saliva tendría una función crítica en la transmisión de SARS-CoV-2 en la población. Es por esto, que los diagnósticos salivales no invasivos podrían proporcionar una plataforma de detección rápida, temprana y poco invasiva de la infección por COVID-19. El objetivo de esta revisión es determinar los beneficios de la saliva como muestra no invasiva para el diagnóstico de COVID-19.
\end{abstract}

PALABRAS CLAVE: Saliva; diagnóstico, COVID-19, Odontología.

\section{INTRODUCCIÓN}

El 29 de diciembre del año 2019, un brote de neumonía fue notificado en la ciudad de Wuhan, China, donde se identificaron 27 casos sin una etiología aparente (Rodriguez-Morales et al., 2020). Actualmente, las secuencias genómicas han sugerido que la emergencia viral está relacionada a los coronavirus de murciélago, pero a diferencia de las infecciones por coronavirus en humanos causadas por betacoronavirus del síndrome respiratorio agudo severo (SARS-CoV) o del síndrome respiratorio del Medio Oriente (MERS-CoV), ha presentado una menor tasa de mortalidad (Sabino-Silva et al., 2020). Sin embargo, se extendió de forma rápida a ciudades y países vecinos, siendo declarada el 30 de enero de 2020 por la OMS como una emergencia de salud pública de preocupación internacional (Sohrabi et al., 2020). El agente causal se identificó a partir de muestras de hisopos de garganta realizadas por el Centro
Chino para el Control y la Prevención de Enfermedades el 7 de enero de 2020 siendo denominado Coronavirus 2 del Síndrome Respiratorio Agudo Severo (SARS-CoV-2). Posteriormente, la enfermedad fue nombrada COVID-19 por la Organización Mundial de la Salud (OMS) (Singhal et al., 2020).

Los estudios han demostrado que los Coronavirus pueden transmitirse de persona a persona a través del contacto directo o indirecto, mediante gotas gruesas o pequeñas de secreciones provenientes del tracto respiratorio (Peng et al., 2020), esto es de vital importancia a nivel odontológico dado que los entornos de atención dental conllevan a riesgos elevados de infección por COVID-19 debido a la especificidad de sus procedimientos, lo que implica contacto cercano con los pacientes asociado a la exposición frecuente a saliva y otros fluidos de ries-

\footnotetext{
${ }^{1}$ Cirujano Dentista, Pasante Servicio de Cirugía Maxilofacial, Hospital San Juan de Dios, Santiago, Chile.

${ }^{2}$ Cirujana Dentista, Facultad de Odontología, Universidad de Chile, Chile.

${ }^{3}$ Cirujano Maxilofacial, Servicio de Cirugía Maxilofacial, Hospital San Juan de Dios, Santiago, Chile.
} 
go (Peng et al.). De hecho, se sabe que el SARSCoV se puede detectar en la saliva a títulos altos (To et al., 2020a), por lo que SARS-CoV-2 podría seguir esta misma tendencia. La inhalación de partículas en el aire y aerosoles producidos durante los procedimientos dentales en pacientes con COVID-19 puede ser un procedimiento de alto riesgo en el que los dentistas están directa y estrechamente expuestos a este virus. Es por esto, que los dentistas son uno de los profesionales de salud más expuestos al contagio de la enfermedad y es crucial que los mismos definan estrategias preventivas para evitar la infección por COVID-19 haciendo énfasis en el posicionamiento del paciente, la higiene de las manos, el uso de equipo de protección estándar y específica al realizar procedimientos de generación de aerosoles. Esto último otorga un rol fundamental a los profesionales encargados de la salud oral en la protección contra la propagación de esta enfermedad, brindando la oportunidad de determinar si un diagnóstico no invasivo de saliva para COVID-19 podría ayudar a detectar dichos virus y reducir su propagación (Sabino-Silva et al.).

\section{Actuales métodos de diagnóstico de COVID-19}

El diagnóstico de COVID- 19 puede efectuarse mediante una combinación de información epidemiológica, síntomas clínicos, hallazgos radiológicos y pruebas de laboratorio (Meng et al., 2020). Se define paciente sospechoso a aquel que presenta signos y síntomas característicos; con antecedentes de viajes a países con riesgo de transmisión local persistente como países asiáticos y países con grandes brotes de la enfermedad como Estados Unidos, España, Brasil e Italia, aunque hoy en día la infección ya se transmitió a más de 150 países en todo el mundo (Meng et al.). También es sospechoso aquel que haya tenido contacto con pacientes con antecedentes de viajes similares o aquellos con infección confirmada por COVID-19 (Singhal et al.). Sin embargo, un caso solo puede ser confirmado con una prueba molecular positiva.

Dentro de las manifestaciones clínicas de esta enfermedad, se ha reportado fiebre, tos seca y disnea como las más prevalentes, seguido de mialgia, esputo, dolor de garganta, dolor de cabeza y diarrea (Rodriguez-Morales et al.). Pudiendo evolucionar como una infección sistémica severa desencadenando cuadros de insuficiencia renal aguda y síndrome de dificultad de respiratoria aguda en personas con múltiples comorbilidades de base (Singhal et al.). A pesar de esta tendencia, se han informado casos asintomáticos, casos sin presencia de fiebre e incluso se han reportado transmisiones antes de la aparición de signos y síntoma, de hecho, estudios han demostrado que el $41,6 \%$ de los casos con COVID19 son asintomáticos (Nishiura et al., 2020), por lo que no es posible realizar un diagnóstico únicamente en base a un análisis clínico (Meng et al.; SabinoSilva et al.).

Todo paciente sospechoso requiere de solicitud de exámenes de laboratorio completos. La infección por COVID-19 puede producir aumento en los niveles proteína $\mathrm{C}$ reactiva, aumento en los niveles de velocidad de sedimentación globular, lactato deshidrogenasa, creatinina y un tiempo prolongado de protrombina (Rodríguez-Morales et al.). Desde el inicio de la pandemia y gracias a la publicación del genoma viral, según la última directriz de diagnóstico y tratamiento de la neumonitis causada por SARSCoV-2 publicada por el gobierno de China, se ha utilizado la prueba de reacción en cadena de la polimerasa con transcriptasa inversa (RT-PCR) para la detección del material genético del virus y como parte de su diagnóstico específico de COVID-19 (Corman et al., 2020). Este material genético es detectado mediante el estudio de muestras del tracto respiratorio recolectadas por hisopos nasofaríngeos u orofaríngeos, esputos, aspirados endotraqueales y lavados broncopulmonares (Sabino-Silva et al.; Cheng et al., 2020), consideradas como muestras del tracto respiratorio inferior; desafortunadamente estudios han demostrado que el solo $28 \%$ de los pacientes con COVID-19 podrían producir un esputo que sirva para diagnóstico de la enfermedad (Sabino-Silva et al.; Toa et al. 2020). Por otra parte, también se ha detectado muestras virales en la sangre y en las heces fecales en cuadros más graves de la enfermedad (Meng et al.). A pesar de que RTPCR es una prueba diagnóstica efectiva, no siempre logra un diagnóstico certero, dado que múltiples factores pueden afectar el resultado de esta prueba como: un muestreo clínico incorrecto, la fuente de muestras (tracto respiratorio superior o inferior), el tiempo de la muestra (período diferente del desarrollo de la enfermedad), el rendimiento de los kits de detección, baja carga viral del paciente (Ai et al., 2020; Fang et al., 2020b). Es por esto que un solo resultado negativo de la prueba de RT-PCR de pacientes sospechosos no excluye la infección por COVID-19, de hecho, se ha informado en estudios que la tasa positiva total de RT-PCR para muestras de hisopos de garganta era de aproximadamente 30 
$\%$ a $60 \%$ (Ai et al.); la baja sensibilidad de RT-PCR implica que muchos pacientes con COVID-19 pueden no ser identificados, pudiendo seguir siendo fuentes de contagio $v$ y no recibir el tratamiento adecuado a tiempo (Fang et al., 2020b).

El uso de tomografía computarizada (TC) de tórax es una alternativa fácil de realizar y con buenos resultados diagnósticos según los últimos estudios publicados; dado que ha mostrado características radiológicas típicas en pacientes con COVID-19 dentro de las cuales se observan aspecto de vidrio esmerilado, cambios en el intersticio pulmonar con distribución periférica y conformación de opacidades multifocales a nivel del pulmón (Ai et al.). Estos signos se identificaron también en pacientes asintomáticos, incluso se ha utilizado para el diagnóstico de COVID19 en pacientes con características epidemiológicas y clínicas compatibles con la enfermedad cuando las pruebas en base a PCR -RT son negativas (Singhal et al.). Estudios recientes han revelado mayor sensibilidad del TC de tórax en relación al RT- PCR, con un 98 $\%$ vs un $71 \%$ respectivamente (Fang et al., 2020a).

\section{Saliva como posible método de diagnóstico de COVID-19}

Se han reportado tres vías diferentes por las cuales el COVID-19 puede presentarse en la saliva. En primer lugar, el virus comúnmente se encuentra en el tracto respiratorio inferior y superior ingresando y relacionandose con la cavidad oral a traves del traspaso de secreciones de manera bidireccional. En segundo lugar, el virus circulante en el torrente sanguíneo puede acceder a la boca a través del líquido gingival crevicular, un exudado específico de la cavidad oral que contiene proteínas locales derivadas de la matriz extracelular y proteínas derivadas del suero (Sabino-Silva et al.). Finalmente, la tercera forma en que el COVID-19 puede presentarse en la cavidad oral es mediante la colonización e infección de las glándulas salivales mayores y menores con la posterior liberación de partículas en la saliva a través de los conductos salivales (Liu et al., 2017). Con respecto a esto último, se ha estudiado qué SARS-CoV-2 ingresa a las células al igual que el coronavirus del SARS, es decir, a través del receptor celular ACE2. Se encontró que las células con receptores ACE2 se distribuyen de forma abundante en todo el tracto respiratorio, así como las células epiteliales de los conductos de las glándulas salivales (Liu et al.). Además, se ha estudiado que las células epiteliales de los conductos salivales que presentan el receptor ACE2 son los prin- cipales objetivos de la infección por el tipo SARS-CoV, y es probable que SARS-CoV-2 sea la misma situación, aunque hasta ahora no se ha informado ningún reporte en relación a esto. (Peng et al.)

La orofaringe reúne las secreciones provenientes de la nasofaringe, las glándulas salivales y las secreciones respiratorias arrastradas desde el árbol traqueal-bronquial. Las pruebas de saliva podrían mostrar desprendimiento viral tanto de las glándulas salivales como del tracto respiratorio superior e inferior (Liu et al.; Sabino-Silva et al.). En adición a lo anteriormente mencionado, estudios en modelos animales que fueron inmunizados vía nasal demostraron la producción de inmunoglobulina A secretora específica (slgA) para el virus SARS-CoV en la saliva (SabinoSilva et al.). Estudios anteriores demostraron que la saliva posee una alta tasa de concordancia con más del $90 \%$ con muestras nasofaríngeas en la detección de virus respiratorios, incluidos los coronavirus, de hecho, se ha reportado que, en algunos pacientes, el coronavirus se detectó solo en la saliva, pero no en muestras de aspirados nasofaríngeos (To et al., 2017). Wang et al. (2004) demostraron una alta carga viral con presencia de ARN del virus SARS-CoV en muestras orofaríngeas y de saliva, en este se usó el ensayo cuantitativo en tiempo real de la RT-PCR para investigar la carga de SARS-CoV en las muestras de saliva, lo que reafirma la hipótesis de usar la saliva como un posible reservorio diagnóstico para la epidemia actual de COVID-19.

Otro estudio pionero realizado en el Hospital Central de Xiangtan analizó los tiempos de conversión de ácido nucleico de SARS-CoV-2 de diferentes muestras obtenidas de pacientes con COVID 19, las cuales fueron sometidas a RT-PCR. La tasa de conversión positiva de ácido nucleico para la saliva fue del $78,1 \%$, siendo muy superior a otras muestras como las lágrimas y la orina; además el tiempo de conversión promedio aproximado fue entre 13 y 17 días (Fang et al., 2020a). Del mismo modo se realizó una evaluación de muestras de saliva orofaríngeas en donde el ARN del SARS-CoV-2 fue detectado en el $87 \%$ de pacientes, además se demostró que la carga viral fue más alta durante la primera semana del inicio de los síntomas y fue decreciendo gradualmente con las semanas siguientes, aunque hubo un caso donde se detectó ARN viral 25 días después del inicio de los síntomas (To et al., 2020b). Con relación a esto, otro estudio realizado en Hong Kong por la subdivisión de Servicios de Laboratorio de Salud Pública, estudiaron 12 pacientes con infección por COVID-19 confirmada; 
tomándose muestras salivales de cada paciente las cuales fueron sometidas posteriormente a análisis mediante RT-PCR y a cultivos virales. El RT-PCR demostró que SARS-CoV-2 pudo detectar material genético viral en 11 de los 12 pacientes estudiados, además las muestras de saliva en serie mostraron disminuciones en los niveles de ARN salival de COVID19 después de la hospitalización (To et al., 2020a). Es por esto, que los biomarcadores que usan saliva podrían mejorar la detección de la enfermedad, siendo una plataforma no invasiva para los pacientes (To et al., 2020a; Sabino-Silva et al.).

\section{Ventajas del uso de saliva}

Existen varias ventajas en el uso de muestras de saliva para el diagnóstico de COVID-19 (Tabla I). El paciente puede proporcionar fácilmente muestras de saliva sin ningún tipo de procedimiento invasivo, a diferencia de los hisopos nasofaríngeos y orofaríngeos, que, a pesar de ser efectivos, son métodos dolorosos e incómodos para el paciente, los cuales pueden generar complicaciones durante la toma de la muestra como hematomas, erosiones de la mucosa y sangrado; situación problemática para aquellos pacientes con enfermedades hemorrágicas tales como trombocitopenia (Khurshid et al., 2020; Sabino-Silva et al.).

Es fundamental el monitoreo en serie de cargas virales, dado que se ha reportado casos con resolución completa de los síntomas, que dieron positivo por SARS-CoV-2 nuevamente después de 2 días de hallazgos negativos, sugiriendo que SARS-CoV-2 podría excretarse a niveles bajos a pesar de la recuperación clínica (To et al., 2020b). Bajo esta misma línea el uso de hisopos nasofaríngeos y orofaríngeos no serían los más adecuados en caso de necesitar controlar series de cargas virales repetidas en comparación con las muestras de saliva (Khurshid et al.), dado que la recolección de estos tipos de muestras requiere personal de salud y un contacto cercano entre los trabajadores de la salud con los pacientes, lo que plantea un riesgo de transmisión del virus a los trabajadores de la salud, mientras que la recolección de saliva podría efectuarse incluso por el mismo paciente (To et al., 2020a). El uso de saliva podría permitir la recolección de muestras fuera de centros hospitalarios, como en clínicas ambulatorias o en centros de atención primaria, que son lugares donde existe un gran número de individuos que requieren detección y donde la saliva representaría un tipo de muestra práctica no invasiva, disminuyendo así el riesgo de transmisión nosocomial de COVID 19 (To et al., 2020a). Por otro lado, el uso de muestras de saliva permitiría ahorrar el tiempo de espera para la recolección de muestras y, por lo tanto, agilizar la disponibilidad de los resultados (To et al., 2020a). Las muestras de saliva se pueden proporcionar fácilmente solicitando a los pacientes que escupan en un frasco estéril, de hecho, existen muchos dispositivos de recolección de saliva disponibles en el mercado para una recolección segura y estéril sin comprometer la calidad y la cantidad (Khurshid et al.).

\section{CONCLUSIÓN}

La saliva tiene un papel fundamental en la transmisión de COVID-19 en la población, de hecho, estudios actuales han demostrado que la saliva podría ser una alternativa validada no invasiva para el diagnóstico y el monitoreo de la carga viral de SARS-CoV-2, proporcionando una plataforma rentable y conveniente.

En conclusión, es fundamental que los centros de investigación a nivel mundial consideren los beneficios de este fluido, así como la posibilidad de identificar marcadores que permitan realizar el diagnóstico de manera segura y poco invasiva.

Tabla I. Resumen comparativo de diagnóstico mediante saliva vs métodos convencionales de diagnóstico de COVID-19.

\begin{tabular}{lll}
\hline & Diagnóstico Salival & Diagnóstico convencional \\
\hline Toma de muestra & Expulsar saliva en recipiente & Hisopos oronasales - orofaríngeos y \\
& & lavados broncopulmonares. \\
Lugar de toma de muestra & Ambiente extrahospitalario y hospitalario & Ambiente hospitalario \\
Riesgo de contaminación & Bajo riesgo & Elevado riesgo \\
Transmisión nosocomial & Bajo riesgo & Elevado riesgo \\
Monitoreo en serie carga viral & Adecuado & Inadecuado \\
Personal de toma de muestra & Puede ser recolectado por el mismo & Requiere personal calificado \\
Complicaciones & No reportadas & Sangrado, desgarros y hematomas \\
\hline Tiempo de recolección & Rápida & Lenta \\
\hline
\end{tabular}


MELIÁN, R. A.; CALCUMIL, H. P.; BOIN, B. C. \& CARRASCO, S. R. Detection of COVID-19 (SARS-CoV-2) by saliva: A low-invasive diagnostic alternative. Int. J. Odontostomat., 14(3):316-320, 2020.

ABSTRACT: The new coronavirus disease, also called COVID 19, is the latest infectious disease of international concern. Originating in Wuhan, China, it spread globally, resulting in the 2019-2020 pandemic and a Public Health Emergency of International Concern (ESPII) as declared by the World Health Organization (WHO). This disease usually presents with fever, cough, sore throat, respiratory distress, fatigue and general discomfort; however, asymptomatic cases have been reported. The diagnosis is made with a combination of clinical, radiological and laboratory molecular tests, where the reverse transcriptase polymerase chain reaction (RT-PCR) test has been the alternative of choice for the analysis of viral genetic material from samples taken of upper respiratory tract. COVID-19 has been reported to be transmitted person-to-person or by indirect fluids drop contact, which is of fundamental importance for clinical dental procedures and where saliva would play a critical role in the transmission of SARS-CoV-2 from human to human. For this reason, non-invasive salivary diagnoses could provide a platform for rapid, early and non-invasive detection of COVID19 infection. The objective of this review is to determine the benefits of saliva as a non-invasive sample for the diagnosis of COVID-19. dentistry.

KEY WORDS: Saliva, diagnosis, COVID-19,

\section{REFERENCIAS BIBLIOGRÁFICAS}

Ai, T.; Yang, Z.; Hou, H.; Zhan, C.; Chen, C.; Lv, W.; Tao, Q.; Sun, Z. \& Xia, L. Correlation of Chest CT and RT-PCR Testing in Coronavirus Disease 2019 (COVID-19) in China: A Report of 1014 Cases. Radiology, 200642, 2020. DOI: https://www.doi.org/10.1148/ radiol.2020200642

Cheng, V. C. C.; Wong, S. C.; Chen, J. H. K.; Yip, C. C. Y.; Chuang, V. W. M.; Tsang, O. T. Y.; Sridhar, S.; Chan, J. F. W.; Ho, P. L. \& Yuen, K. Y. Escalating infection control response to the rapidly evolving epidemiology of the coronavirus disease 2019 (COVID-19) due to SARS-CoV-2 in Hong Kong. Infect. Control Hosp. Epidemiol., 2020. DOI: https://www.doi.org/10.1017/ice.2020.58

Corman, V. M.; Landt, O.; Kaiser, M.; Molenkamp, R.; Meijer, A.; Chu, D. K.; Bleicker, T.; Brünink, S.; Schneider, J.; Schmidt, M. L.; et al. Detection of 2019 novel coronavirus (2019-nCoV) by real-time RTPCR. Euro Surveill., 25(3), 2020. DOI: https://www.doi.org/10.2807/ 1560-7917.

Fang, Y.; Zhang, H.; Xie, J.; Lin, M.; Ying, L.; Pang, P. \& Ji, W. Sensitivity of Chest CT for COVID-19: Comparison to RT-PCR. Radiology, 200432, 2020a. DOI: https://www.doi.org/10.1148/ radiol.2020200432

Fang, Z.; Zhang, Y.; Hang, C.; Li, S.; Ai, J. \& Zhang, W. Comparisons of viral shedding time of SARS-CoV-2 of different samples in ICU and non-ICU patients. J. Infect., 2020b. DOI: https://www.doi.org/ 10.1016/j.jinf.2020.03.013
Khurshid, Z.; Asiri, F. Y. I. \& AI Wadaani, H. Human Saliva: Non-Invasive Fluid for Detecting Novel Coronavirus (2019-nCoV). Int. J. Environ. Res. Public Health, 17(7):2225, 2020. DOI: https://www.doi.org/ 10.3390/ijerph17072225

Meng, L.; Hua, F. \& Bian, Z. Coronavirus Disease 2019 (COVID-19): Emerging and Future Challenges for Dental and Oral Medicine. J. Dent. Res., 99(5):481-7, 2020. DOI: https://www.doi.org/10.1177/ 0022034520914246

Nishiura, H.; Kobayashi, T.; Suzuki, A.; Jung, S. M.; Hayashi, K.; Kinoshita, R.; Yang, Y.; Yuan, B.; Akhmetzhanov, A. R.; Linton, N M.; et al. Estimation of the asymptomatic ratio of novel coronavirus infections (COVID-19). Int. J. Infect. Dis., 2020. DOI: https:// www.doi.org/10.1016/j.ijid.2020.03.020

Peng, X.; Xu, X.; Li, Y.; Cheng, L.; Zhou, X. \& Ren, B. Transmission routes of 2019-nCoV and controls in dental practice. Int. J. Oral Sci., 12:9, 2020. DOI: https://www.doi.org/10.1038/s41368-0200075-9

Rodriguez-Morales, A. J.; Cardona-Ospina, J. A.; Gutiérrez-Ocampo, E.; Villamizar-Peña, R.; Holguin-Rivera, Y.; Escalera-Antezana, J. P.; Alvarado-Arnez, L. E.; Bonilla-Aldana, D. K.; Franco-Paredes, C.; Henao-Martinez, A. F.; et al. Clinical, laboratory and imaging features of COVID-19: A systematic review and meta-analysis. Travel Med. Infect. Dis., 101623, 2020. DOI: https://www.doi.org/ 10.1016/j.tmaid.2020.101623

Sabino-Silva, R.; Jardim, A. C. G. \& Siqueira, W. L. Coronavirus COVID19 impacts to dentistry and potential salivary diagnosis. Clin. Oral Investig., 24(4):1619-21, 2020. DOI: https://www.doi.org/10.1007/ s00784-020-03248-x

Singhal, T. A Review of Coronavirus Disease-2019 (COVID-19). Indian J. Pediatr., 87:281-6, 2020. DOI: https://www.doi.org/10.1007/ s12098-020-03263-6

Sohrabi, C.; Alsafi, Z.; O'Neill, N.; Khan, M.; Kerwan, A.; Al-Jabir, A.; Iosifidis, C. \& Agha, R. World Health Organization declares global emergency: A review of the 2019 novel coronavirus (COVID-19). Int. J. Surg., 76:71-6, 2020.

To, K. K.; Tsang, O. T.; Leung, W. S.; Tam, A. R.; Wu, T. C.; Lung, D. C.; Yip, C. C.; Cai, J. P.; Chan, J. M.; Chik, T. S.; et al. Temporal profiles of viral load in posterior oropharyngeal saliva samples and serum antibody responses during infection by SARS-CoV-2: an observational cohort study. Lancet Infect. Dis., 2020b. DOI: https:/ /www.doi.org/10.1016/S1473-3099(20)30196-1

To, K. K.; Tsang, O. T.; Chik-Yan Yip, C.; Chan, K. H.; Wu, T. C.; Chan, J. M. C.; Leung, W. S.; Chik, T. S.; Choi, C. Y.; Kandamby, D. H.; et al. Consistent detection of 2019 novel coronavirus in saliva. Clin. Infect. Dis., ciaa149, 2020a. DOI: https://www.doi.org/10.1093/cid/ ciaa149

To, K. K.; Lu, L.; Yip, C. C.; Poon, R. W.; Fung, A. M.; Cheng, A.; Lui, D. H.; Ho, D. T.; Hung, I. F.; Chan, K. H.; et al. Additional molecular testing of saliva specimens improves the detection of respiratory viruses. Emerg. Microbes Infect.. 6(6):e49, 2017.

Wang, W. K.; Chen, S. Y.; Liu, I. J.; Chen, Y. C.; Chen, H. L.; Yang, C. F.; Chen, P. J.; Yeh, S. H.; Kao, C. L.; Huang, L. M.; et al. Detection of SARS-associated coronavirus in throat wash and saliva in early diagnosis. Emerg. Infect. Dis., 10(7):1213-9, 2004

Autor para correspondencia:

Andrés Melián Rivas

Hospital San Juan de Dios

AV. Portales 3239

Santiago

CHILE

Recibido: 21-04-2020

Aceptado: 22-04-2020

Correo: andresmelianrivas@yahoo.es 